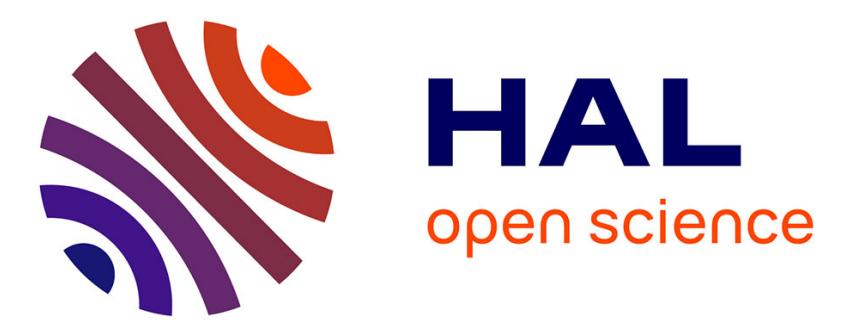

\title{
Multi-Compartment Model of Brain Tissues from T2 Relaxometry MRI Using Gamma Distribution
}

Sudhanya Chatterjee, Olivier Commowick, Onur Afacan, Simon K Warfield, Christian Barillot

\section{To cite this version:}

Sudhanya Chatterjee, Olivier Commowick, Onur Afacan, Simon K Warfield, Christian Barillot. MultiCompartment Model of Brain Tissues from T2 Relaxometry MRI Using Gamma Distribution. ISBI 2018 - IEEE International Symposium on Biomedical Imaging, Apr 2018, Washington DC, United States. pp.141-144, 10.1109/ISBI.2018.8363541 . hal-01744852

\section{HAL Id: hal-01744852 https://hal.science/hal-01744852}

Submitted on 27 Mar 2018

HAL is a multi-disciplinary open access archive for the deposit and dissemination of scientific research documents, whether they are published or not. The documents may come from teaching and research institutions in France or abroad, or from public or private research centers.
L'archive ouverte pluridisciplinaire HAL, est destinée au dépôt et à la diffusion de documents scientifiques de niveau recherche, publiés ou non, émanant des établissements d'enseignement et de recherche français ou étrangers, des laboratoires publics ou privés. 


\title{
MULTI-COMPARTMENT MODEL OF BRAIN TISSUES FROM T2 RELAXOMETRY MRI USING GAMMA DISTRIBUTION
}

\author{
Sudhanya Chatterjee ${ }^{\star}$ Olivier Commowick ${ }^{\star}$ Onur Afacan ${ }^{\dagger}$ Simon K. Warfield Christian Barillot $^{\star}$ \\ ${ }^{\star}$ VisAGeS U1228 INSERM/Inria, IRISA UMR CNRS 6074, University of Rennes 1, France \\ ${ }^{\dagger}$ Computational Radiology Laboratory, Boston Children's Hospital, Boston, MA, USA
}

\begin{abstract}
The brain microstructure, especially myelinated axons and free fluids, may provide useful insight into brain neurodegenerative diseases such as multiple sclerosis (MS). These may be distinguished based on their transverse relaxation times which can be measured using $T_{2}$ relaxometry MRI. However, due to physical limitations on achievable resolution, each voxel contains a combination of these tissues, rendering the estimation complex. We present a novel multi-compartment $T_{2}$ (MCT2) estimation based on variable projection, applicable to any MCT2 microstructure model. We derive this estimation for a three-gamma distribution model. We validate our framework on synthetic data and illustrate its potential on healthy volunteer and MS patient data.
\end{abstract}

Index Terms $-T_{2}$ relaxometry, microstructure, brain

\section{INTRODUCTION}

MRI voxels of the human brain are heterogeneous in terms of tissue types due to the limited imaging resolution and physical constraints. Each voxel in the white matter (WM) contains a large number of myelinated and non-myelinated axons, glial cells and extracellular fluids [1,2]. For example, every square millimeter of the corpus callosum in a human brain has more than 100,000 fibers (myelinated and non-myelinated) of varying diameters [1]. These tissues can be distinguished based on their $T_{2}$ relaxation times. Myelin being a tightly wrapped structure has a very short $T_{2}$ relaxation time of 10 milliseconds $(m s)$ [2]. The estimated $T_{2}$ relaxation time of the myelinated axons is $40 \mathrm{~ms}[2]$. The ventricles and tissue injury regions contain free fluids which have a high $T_{2}$ relaxation time ( $>1000 \mathrm{~ms}$ ). The $T_{2}$ relaxation values between those of myelin and myelinated axons and the free fluids correspond to the glial cells and extracellular tissues [2]. An ability to obtain the condition of these tissues can help us gain better insights into the onset and progress of neurodegenerative diseases such as multiple sclerosis (MS).

Myelin water fraction (MWF) has been computed from $T_{2}$ relaxometry images using a variety of approaches [3, 4]. Most of these methods primarily focus on the MWF estimation. However, MWF alone might not be able to convey the en- tire information since it is a relative measurement. For example, in MS patients a decrease in MWF in a WM lesion might be caused by myelin loss or fluid accumulation due to tissue injury or both. Hence for relative measurements like water fraction (WF), all the WF maps should be observed simultaneously for a complete understanding of the tissue condition. Here we propose an estimation framework to obtain brain microstructure information using a multi-compartment tissue model from $T_{2}$ relaxometry MRI data. The $T_{2}$ space is modeled as a weighted mixture of three continuous probability density functions (PDF), each representing the tissues with short, medium and high $T_{2}$ relaxation times. We estimate the PDF parameters using variable projection (VARPRO) approach [5]. We derive this generic estimation framework for gamma PDFs. We validate the proposed method using synthetic data against known ground truth. We then illustrated it on a healthy subject and MS patient.

\section{METHOD AND MATERIALS}

\subsection{Theory}

Signal model The $T_{2}$ space is modeled as a weighted mixture of three PDFs, representing each of the three $T_{2}$ relaxometry compartments: short-, medium - and high $-T_{2}$. Thus the voxel signal at the $i$-th echo time $\left(t_{i}\right)$ is given as:

$s\left(t_{i}\right)=\mathrm{M}_{0} \sum_{j=1}^{3} w_{j} \int_{0}^{\infty} f_{j}\left(T_{2} ; \mathbf{p}_{\mathbf{j}}\right) \operatorname{EPG}\left(T_{2}, \triangle T E, i, B_{1}\right) d T_{2}$

Each compartment is described by a chosen PDF, $f_{j}\left(T_{2} ; \mathbf{p}_{\mathbf{j}}\right)$, where $\mathbf{p}_{\mathbf{j}}=\left\{p_{j_{1}}, \ldots, p_{j_{n}}\right\} \in \mathbb{R}^{n}$ are the PDF parameters. In Eq. (1), $w_{j}$ is the weight of the $j$-th distribution with $\sum_{j} w_{j}=1 . \Delta T E, B_{1}$ and $M_{0}$ are the echo spacing, field inhomogeneity and magnetization constant respectively. Imperfect rephasing of the nuclear spins after application of refocusing pulses leads to the generation of stimulated echoes [6]. Hence the $T_{2}$ decay is not purely exponential. The stimulated echoes are thus obtained using the EPG algorithm [7]. $\mathrm{EPG}(\cdot)$ is the stimulated echo computed at $t_{i}=i \Delta \mathrm{TE}$ where $i=\{1, \ldots, m\}$ and $m$ is the number of echoes. 
Optimization $M_{0}$ and $w_{j}$ can be combined into a single term $\alpha_{j} \in \mathbb{R}^{+}$without any loss of generality. In that case, the weight $w_{j}$ corresponding to each compartment is obtained as $w_{j}=\alpha_{j} / \sum_{i} \alpha_{i}$. In the most general case, the least squares minimization problem is thus formulated as:

$$
\begin{aligned}
\left(\hat{\alpha}, \hat{\mathbf{p}}, \hat{B_{1}}\right) & =\arg \min _{\alpha, \mathbf{p}, B_{1}} \sum_{i=1}^{m}\left(y_{i}-\sum_{j=1}^{3} \alpha_{j} \lambda_{j}\left(t_{i} ; \mathbf{p}, B_{1}\right)\right)^{2} \\
& =\arg \min _{\alpha, \mathbf{p}, B_{1}}\left\|\mathbf{Y}-\boldsymbol{\Lambda}\left(\mathbf{p}, B_{1}\right) \alpha\right\|_{2}^{2}
\end{aligned}
$$

where $\mathbf{Y} \in \mathbb{R}^{m}$ is the observed signal and $m$ is the number of echoes; $\alpha \in \mathbb{R}^{+^{3}} ; \boldsymbol{\Lambda} \in \mathbb{R}^{m \times 3} ; \mathbf{p}=\left\{\mathbf{p}_{\mathbf{1}}, \mathbf{p}_{\mathbf{2}}, \mathbf{p}_{\mathbf{3}}\right\} \in$ $\mathbb{R}^{k}$, where $k=3 n$. In Eq. (2), each element of $\boldsymbol{\Lambda}$, $\Lambda_{i j}=\lambda\left(t_{i} ; \mathbf{p}_{\mathbf{j}}, B_{1}\right)$, is computed as:

$$
\Lambda_{i j}=\int_{0}^{\infty} f_{j}\left(T_{2} ; \mathbf{p}_{\mathbf{j}}\right) \operatorname{EPG}\left(T_{2}, \triangle T E, i, B_{1}\right) d T_{2}
$$

Due to the EPG formulation, there is no closed form derivative solution for the optimization of $B_{1}$ [7]. Hence, we opt for an alternate optimization scheme where we iterate between optimization of $\{\mathbf{p}, \alpha\}$ with a fixed value of $B_{1}$ and optimization for $B_{1}$ using the obtained $\{\mathbf{p}, \alpha\}$ values. The terms $\boldsymbol{\Lambda}\left(\mathbf{p}, B_{1}\right)$ and $\alpha$ in Eq. (2) are linearly separable. Hence we can use the VARPRO approach to solve for $\{\mathbf{p}, \alpha\}$ [5]. The unknown $\alpha$ is substituted by $\boldsymbol{\Lambda}(\mathbf{p})^{+} \mathbf{Y}$, where $\boldsymbol{\Lambda}(\mathbf{p})^{+}$is the Moore-Penrose generalized inverse of $\boldsymbol{\Lambda}(\mathbf{p})$. The VARPRO cost function is computed as:

$$
\arg \min _{\mathbf{p}}\left\|\left(\mathbf{I}-\boldsymbol{\Lambda}(\mathbf{p}) \boldsymbol{\Lambda}(\mathbf{p})^{+}\right) \mathbf{Y}\right\|_{2}^{2}
$$

where, $\mathbf{I}-\boldsymbol{\Lambda}(\mathbf{p}) \boldsymbol{\Lambda}(\mathbf{p})^{+}$is the projector on the orthogonal complement of the column space of $\Lambda(\mathbf{p})$. Since $\mathbf{p} \in \mathbb{R}^{k}$, the Jacobian matrix $\mathbf{J} \in \mathbb{R}^{k \times m}$ and its columns are computed as shown in [5]. To compute the elements of $\mathbf{J}$, we need to obtain $\partial \Lambda / \partial p_{j_{i}}, \forall i, j$ [5]. After solving Eq. (4) for $\mathbf{p}$, the values of $\alpha$ are obtained as $\Lambda(\mathbf{p})^{+} \mathbf{Y}$. The optimization for $\{\alpha, \mathbf{p}\}$ and $B_{1}$ is performed alternatively until convergence. $\mathrm{B}_{1}$ is optimized using a gradient free optimizer (BOBYQA), as it does not have any closed form solution [7].

Multi-compartment model using gamma PDF The previous estimation framework is generic as it does not depend on the chosen PDF. We choose here to use gamma PDF for $f_{j}(\cdot)$ for $j=\{1,2,3\}$ since their non-negativity and skewed nature are well suited to describe the compartments used to model the $T_{2}$ space. The mean $T_{2}$ values of myelin, myelinated axons, inter- and extra-cellular and free fluids in the brain are well studied in the literature [2, 3]. Hence we parameterized each $f_{j}$ in terms of its mean $\left(\mu_{j}\right)$ and variance $\left(v_{j}\right)$ rather than the usual shape and scale parameter representation (refer Eq. (5)). Using this parametric form of the gamma PDF makes the choice of optimization bounds convenient.

$$
f\left(T_{2} ; \mu_{j}, v_{j}\right)=\frac{T_{2}^{\left(\mu_{j}^{2} / v_{j}\right)-1}}{\Gamma\left(\mu_{j}^{2} / v_{j}\right)\left(v_{j} / \mu_{j}\right)^{\frac{\mu_{j}^{2}}{v_{j}}}} \exp \left(\frac{-T_{2}}{v_{j} / \mu_{j}}\right)
$$

Hence we have, $\mathbf{p}=\left\{\mu_{s}, v_{s}, \mu_{m}, v_{m}, \mu_{h}, v_{h}\right\}$ where, $(\cdot)_{s}$, $(\cdot)_{m}$ and $(\cdot)_{h}$ are the PDF parameters describing the short, medium- and high- $T_{2}$ compartments respectively. Due to practical limitations such as feasible acquisition time, coil heating and specific absorption rate (SAR) guidelines, $T_{2}$ relaxometry MRI sequences have limitations on the shortest echo time and number of echoes per acquisition. The high$T_{2}$ compartment aims at capturing of free fluids in the brain, and hence has a $T_{2}$ relaxation time larger than 1 second [3]. A standard $T_{2}$ spin echo multi-contrast sequence has the shortest $T_{2}$ acquisition (first echo) at around $8-10 \mathrm{~ms}$ and has $20-40$ acquired echoes. Hence for the short- $T_{2}$ compartment, we usually have a very limited (around 3-4) number of echoes. There are almost no echoes available which correspond to the high- $T_{2}$ compartment. The robustness and accuracy of the implementations to simultaneously estimate the weights and all the PDF parameters has been found to be not reliable [8]. Hence we choose to estimate only the mean of the gamma PDF corresponding to the medium- $T_{2}$ compartment.

Using the VARPRO approach we thus estimate four parameters of the signal model: mean of the medium- $T_{2}$ gamma PDF $\left(\mu_{m}\right)$ and the three weights corresponding to each compartment. Hence only $\partial \Lambda / \partial \mu_{m}$ is required for computing the Jacobian matrix and is obtained as:

$$
\begin{aligned}
\frac{\partial \Lambda}{\partial \mu_{m}} & =\int_{0}^{\infty} f\left(T_{2} ; \mu_{m}, v_{m}\right)\left[\frac { \mu _ { m } } { v _ { m } } \left(2 \log \left(T_{2} \frac{\mu_{m}}{v_{m}}\right)-\right.\right. \\
& \left.\left.2 \Psi\left(\frac{\mu_{m}^{2}}{v_{m}}\right)+1\right)-\frac{T_{2}}{v_{m}}\right] \operatorname{EPG}\left(T_{2}, \triangle T E, i, B_{1}\right) d T_{2}
\end{aligned}
$$

where $\Psi(\cdot)$ is the digamma function. The remaining gamma PDF parameter values are pre-selected for the three compartments based on histology findings reported in the literature [3] and are set as $\left\{\mu_{s}, \mu_{h}\right\}=\{30,2000\} \mathrm{ms}$ and $\left\{v_{s}, v_{m}, v_{h}\right\}=$ $\{50,100,6400\} \mathrm{ms}^{2}$. We assume a reasonable bound on $\mu_{m}$ of $100-125 \mathrm{~ms}$ for its optimization. The minimization problem in Eq. (4) is solved for $\mu_{m}$ using the analytically obtained derivative in Eq. (6) with a gradient based optimizer [9]. The short- $T_{2}$ compartment here indicates the condition of myelin and myelinated axons [2]. The medium- $T_{2}$ compartment's WF conveys information on the condition of axons, glial cells and extracellular fluids [2]. The condition of free fluids (such as in ventricles and fluid accumulation due to tissue injuries) is indicated by the high- $T_{2} \mathrm{WF}$ values.

\subsection{Experiments}

Synthetic data. The proposed method was first validated against synthetic data generated following a known ground 
truth, composed of three gamma PDFs with parameters, $\left\{\mu_{s}\right.$, $\left.\mu_{m}, \mu_{h}\right\}=\{25,120,1900\}$ and $\left\{v_{s}, v_{m}, v_{h}\right\}=\{40,90$, $6000\}$. The weights chosen for each compartment were, $\left\{w_{s}\right.$, $\left.w_{m}, w_{h}\right\}=\{0.2,0.7,0.1\}$. The $B_{1}, M_{0}$ and $T_{1}$ values considered for this simulation were 1.3 , 950 and 1000 respectively. The experiments are carried out for SNR values ranging from 5 to 100 in steps of 5 . We simulated $T_{2}$ relaxometry data with the following parameters: first echo $\left(\mathrm{TE}_{0}\right)$ is at $9 \mathrm{~ms} ; \triangle \mathrm{TE}=9 \mathrm{~ms} ; 32$ echoes, 100 signal averages.

Healthy volunteer data. The method was tested on $T_{2}$ relaxometry data acquired on a healthy volunteer (male, age: 26) with the following acquisition details: Siemens 3T MRI scanner; 2D multislice CPMG sequence; 32 echoes; $\mathrm{TE}_{0}=9 \mathrm{~ms}$; echo spacing of $9 \mathrm{~ms} ; \mathrm{TR}=3000 \mathrm{~ms}$; single slice acquired; slice thickness of $4 \mathrm{~mm}$; in plane resolution of $1.04 \mathrm{~mm} \times 1.04 \mathrm{~mm}$; matrix size of $192 \times 192$.

MS patient data. The method was finally tested on $T_{2}$ relaxometry MRI data of a MS patient. The observations from our estimation maps were compared with the pathological findings on MS lesion reported in the literature [10, 11]. We observed whether the estimation maps obtained from our method were able to provide insight into MS lesion which corroborate with the pathological findings. The acquisition details are the same as for the healthy volunteer data.

\section{RESULTS}

Synthetic data. The results of the synthetic data simulation are shown in Fig 1. It shows that with increasing SNR the weights estimation gets more accurate. The bars around the mean value are the $95 \%$ confidence intervals (CI) which is obtained as 1.96 times the standard deviation of the estimates. The CIs of the estimation improve with with increasing SNR for all three WFs. The ground truth lies in the CI of the mean estimated weights for all three compartments.

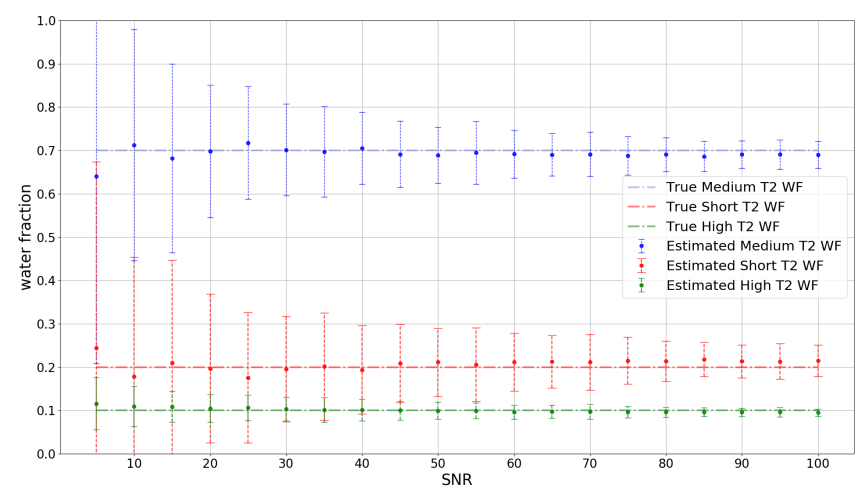

Fig. 1. Mean of estimated weights with a $95 \%$ confidence interval for 100 signal averages for the synthetic data study.

Healthy volunteer data. The estimation maps for the healthy volunteer are shown in Fig 2. The genu of the corpus callosum $(\mathrm{CC})$ has higher $w_{s}$ values compared to any other region in the brain. The ventricles and other regions with free fluids have a higher $\mu_{m}$ compared to the normal appearing white matter (NAWM) tissues. This is relevant as free fluids have a higher $T_{2}$ value compared to the relatively tightly bound tissues present in NAWM.

MS patient data. Two lesions are present in the MR image of the MS patient shown in Fig. 3 that are marked with red and blue arrows. We observe absence of short- $T_{2}$ WF in both lesions. The lesions and their neighboring tissues have higher $w_{m}$ values than NAWM tissues. The $w_{h}$ map shows fluid accumulation in lesion-2 but not in lesion-1. The estimated medium- $T_{2}$ gamma PDF map shows a higher PDF mean for both lesions compared to the NAWM. In lesion-1 the estimated $\mu_{m}$ increases (with respect to the neighboring NAWM) as it approaches the core of the lesion, but is less than the $\mu_{m}$ estimated at the ventricles where there is free fluid.

\section{DISCUSSION}

Our method was successfully validated against synthetic data with known ground truth for all SNR values (refer Fig. 1). High $w_{s}$ values in the genu of the $\mathrm{CC}$ of healthy volunteer data (refer Fig. 2) is due to the high myelin and myelinated fibers density in this region compared to any other part of the brain [1]. In the estimation maps of the MS patient (refer Fig. 3), the absence of short- $T_{2} \mathrm{WF}$ in the lesions and in its neighboring regions can be explained by demyelination of the nerve fibers caused by MS [10][3]. Demyelination at the onset of MS is followed by macrophage intervention leading to an increased cellular activity in the MS lesion regions [10] whose $T_{2}$ relaxation time is greater than myelin and myelinated axons but less than those of free fluids [2]. This phenomenon might explain the high $w_{m}$ values in the lesions and in neighboring regions. Demyelination is followed by progressive axonal damage and fluid accumulation (due to tissue injuries) in MS lesions [10]. The extent of axonal damage and fluid accumulation in the lesions can provide useful information regarding the lesion state and its response to a treatment. Lesion-2 has fluid accumulation unlike lesion-1, possibly indicating that the two lesions are in different stages. The continuous axonal damage in the MS lesions [10][11] explains the higher $\mu_{m}$ in the lesion regions compared to the neighboring NAWM as a higher $\mu_{m}$ value indicates tissues with less tightly bound water. The increment in $\mu_{m}$ values in lesion1 as we approach the lesion core from the lesion boundary might also indicate a reduction in axon density. This is in accordance with the pathology of MS lesion evolution [10][11].

\section{CONCLUSION}

We proposed a generic estimation method to obtain estimates of tissue microstructure in brain by modeling the $T_{2}$ spectrum as a weighted mixture of three gamma PDFs. The maps estimated from our method can be effective in understanding 


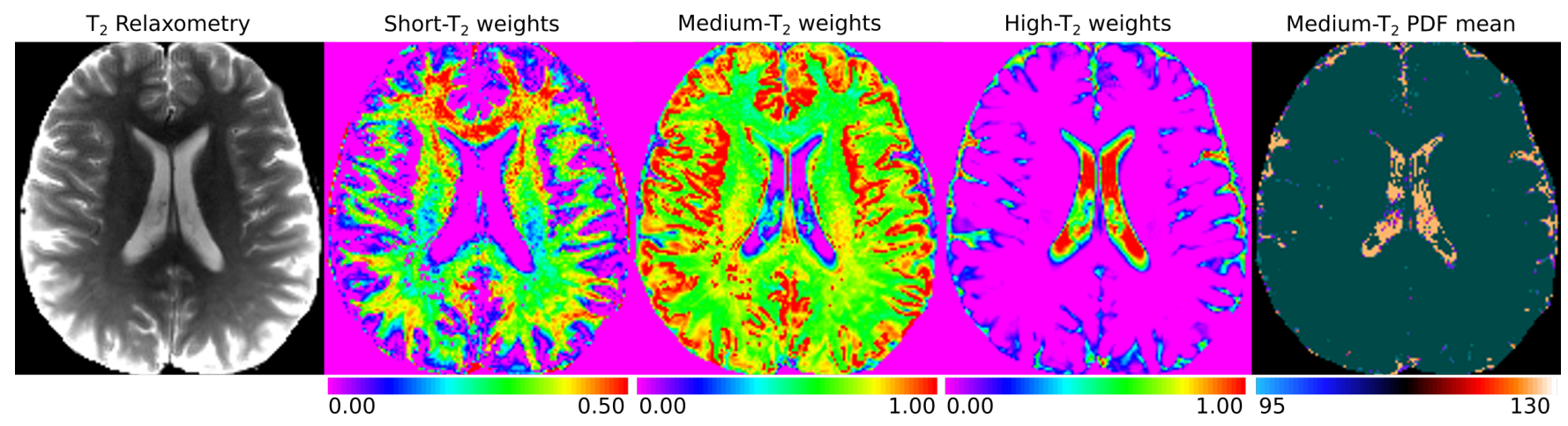

Fig. 2. Estimation maps for a healthy volunteer.

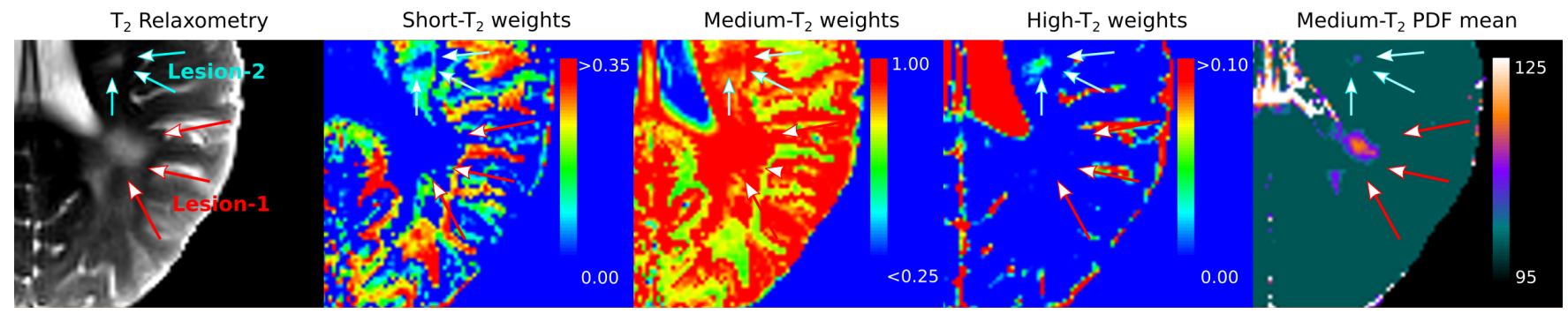

Fig. 3. Estimation maps for MS patient data. Lesion-1 and lesion-2 are marked with red and blue arrows respectively.

the heterogeneity of lesions [10] in MS patients and be used as potential biomarkers to have information on the MS lesion growth stage. As a part of the future study we intend to validate the observations by applying the proposed estimation framework on more healthy controls and MS patient datasets.

\section{REFERENCES}

[1] J Tomasch, "Size, distribution, and number of fibres in the human corpus callosum," The Anatomical Record, vol. 119, no. 1, pp. 119-135, 1954.

[2] JL Lancaster, T Andrews, LJ Hardies, S Dodd, and PT Fox, "Three-pool model of white matter," JMRI, vol. 17, no. 1, pp. 1-10, 2003.

[3] AL MacKay and C Laule, "Magnetic resonance of myelin water: An in vivo marker for myelin," Brain Plasticity, vol. 2, no. 1, pp. 71-91, 2016.

[4] A Akhondi-Asl, O Afacan, M Balasubramanian, RV Mulkern, and SK Warfield, "Fast myelin water fraction estimation using 2D multislice CPMG," MRM, 2015.

[5] G. Golub and V. Pereyra, "Separable nonlinear least squares: the variable projection method and its applications," Inverse Problems, vol. 19, no. 2, pp. R1-R26, 2003.
[6] J Hennig, M Weigel, and K Scheffler, "Calculation of Flip Angles for Echo Trains with Predefined Amplitudes with the Extended Phase Graph (EPG)-Algorithm: Principles and Applications to Hyperecho and TRAPS Sequences," MRM, vol. 51, no. 1, pp. 68-80, 2004.

[7] T Prasloski, B Mädler, QS Xiang, A MacKay, and C Jones, "Applications of stimulated echo correction to multicomponent T2 analysis," MRM, vol. 67, no. 6, pp. 1803-1814, 2012.

[8] KJ Layton, M Morelande, D Wright, PM Farrell, B Moran, and LA Johnston, "Modelling and estimation of multicomponent $T_{2}$ distributions," IEEE TMI, vol. 32, no. 8, pp. 1423-1434, 2013.

[9] K Svanberg, "A class of globally convergent optimization methods based on conservative convex separable approximations," SIAM journal on optimization, vol. 12, no. 2, pp. 555-573, 2002.

[10] H. Lassmann, W. Bruck, and C. Lucchinetti, "Heterogeneity of multiple sclerosis pathogenesis: Implications for diagnosis and therapy," Trends in Molecular Medicine, vol. 7, no. 3, pp. 115-121, 2001.

[11] CR Guttmann, SS Ahn, L Hsu, R Kikinis, and FA Jolesz, "The evolution of multiple sclerosis lesions on serial MR," American journal of neuroradiology, vol. 16, no. 7, pp. 1481-1491, 1995. 